\title{
Introduction to special issue on transportation infrastructure management
}

\author{
Pablo L. Durango-Cohen ${ }^{1} \cdot$ Samer M. Madanat $^{2}$
}

Published online: 19 March 2015

(C) Springer-Verlag Berlin Heidelberg and EURO - The Association of European Operational Research Societies 2015

Transportation infrastructure provides mobility, and thus access to people, goods, services and resources. The availability and level of service of these systems determine quality of life and the continuity of economic and business growth, and, in turn, have served as motivation to develop models to support their management. In the last two decades, there have been significant developments in technologies to collect data related to infrastructure condition, as well as in theoretical and computational capabilities enabling the solution of ever more sophisticated and realistic resource allocation problems. As in other areas, these advances have, in turn, spurred research to improve models, for example to better capture alternatives and their impact (on users), and to address technical issues, such as accounting for serial dependence or unobserved heterogeneity. The motivation for the special issue is to show how researchers from around the world are addressing these issues. Specifically, the special issue includes papers that present interdisciplinary quantitative methods to address problems arising in monitoring, evaluating, repairing, and renewing transportation infrastructure systems with the objective of ensuring that they are capable of performing the functions for which they were designed and built in a reliable, sustainable and equitable fashion.

Samer M. Madanat

madanat@ce.berkeley.edu

Pablo L. Durango-Cohen

pdc@northwestern.edu

1 Department of Civil and Environmental Engineering, Northwestern University, Evanston, IL, USA

2 Department of Civil and Environmental Engineering, University of California, Berkeley, CA, USA 
The call for papers was received enthusiastically, and we received 12 manuscripts from researchers in 7 countries. Eight of these 12 papers are included in the special issue. We feel that, not only the papers are representative of research taking place around the world, but significantly, that they are representative of emerging areas that are relevant to the journal's readership. As an example, we note that three of the papers address problems arising in the management of rail networks, and thus represent a shift from the field's traditional focus on pavement and bridge management.

In the remainder of the section, we provide readers with a brief summary of the papers appearing in the special issue. In terms of organization, the first two papers present econometric models to describe and predict the effect of explanatory factors, including design characteristics, traffic loads and environmental conditions, on the performance/deterioration of infrastructure facilities. The remaining papers address problems of deciding the timing, type and magnitude of interventions aimed at mitigating or reversing the adverse effects of deterioration, such as scheduling maintenance, repair and reconstruction activities.

As stated above, recent technological advances have resulted in the development/ adaptation of sensors and equipment that allow for long-term, continuous monitoring of the condition of (elements that comprise) infrastructure facilities, as well as the factors that contribute to their deterioration. This, in turn, has motivated the formulation and analysis of time series models as a rigorous approach to leverage these newly available longitudinal data sets. The models presented by Kobayashi, Kaito, and Kazumi are the most sophisticated such models presented thus far. Specifically, they propose ARMAX-GARCH models as a framework to capture serial dependence rigorously, and to estimate the effect of exogenous, explanatory variables. The authors also derive a Monte Carlo Markov Chain method to solve the underlying estimation problem. To illustrate the framework, the authors model changes in the long-term vibration profiles, i.e., resonant frequencies, of a set of joints on a viaduct in Japan. To conclude, the authors discuss possible applications of their framework to support maintenance optimization and structural health monitoring.

As in other panel data analyses, the presence of unobserved heterogeneity is a critical issue in the estimation of infrastructure performance models. Medury, Zhang, and Durango-Cohen present mixture regression as a framework to account for heterogeneity. The approach relies on the assumption that the underlying population is comprised of a finite set of classes/segments in unknown proportions. The segmentation basis is latent meaning that the criteria to establish the number and type of segments are related to unobserved heterogeneity manifested in facility performance/deterioration. The segments, in turn, are characterized by a set of commonly specified regression equations, which allows for the identification and estimation of coefficients, i.e., group-level effects, which differ in terms of their level of significance, magnitude or sign. The authors derive an instance of the Expectation-Maximization Algorithm to estimate the associated parameters, and to assign facilities to the population segments, and analyze the performance of a panel of pavements from the AASHO Road Test to illustrate the framework. 
Bai, Gungor, Hernandez-Urrea, Ouyang, and Al-Qadi present a model that shows how characterizations of infrastructure deterioration can be used to support resource allocation. Thus, the paper provides a bridge between the two groups of papers in the special issue. Specifically, the authors use the AASHTOWare Pavement Mechanistic-Empirical Design framework to capture the combined effects of permanent deformation, fatigue, and thermal cracking on pavement deterioration. They then present a mixed-integer nonlinear programming model to optimize rehabilitation timing and magnitude/thickness, with the objective to minimize agency and user costs. The authors propose a solution approach that relies on reformulating the optimization problem as a dynamic program. Numerical examples are used to illustrate the model, and to highlight managerial insights.

The paper by Lee and Madanat present a control model for the problem of optimizing (the timing of) pavement maintenance, rehabilitation and reconstruction. The paper contributes a model where these decisions are considered in an integrated fashion. The authors derive analytical properties of optimal strategies, and present numerical examples to highlight the model's advantages vis-à-vis alternatives that consider the decisions separately.

The papers by Gustavsson, Heinicke, Simroth, Scheithauer, and Fischer, and Savelsbergh, Waterer, Dall, and Moffiet present models to support the management of rail track. Gustavsson presents a mixed-integer linear programming model to schedule tamping operations. The model presented in the paper allows for setup costs, and thus constitutes a generalization to other models appearing in the literature. Numerical examples are used to highlight key advantages of the proposed model over commonly used heuristics. While Gustavsson focuses on costs incurred by agencies, Heinicke et al. consider a situation that includes both agency and user costs. In particular, they consider the problem of assigning maintenance crews to job sequences throughout a rail network. Agencies prefer sequences that minimize travel distance, whereas users prefer sequences where maintenance jobs at critical locations are performed first. The authors show that the resulting scheduling problem is a multi-depot vehicle routing problem with travel and customer costs, and present alternative mixed-integer linear programming formulations to solve the problem. A numerical study is used to compare the formulations. Savelsbergh et al. report on the development of a comprehensive, optimization-based decision-support tool which allows for the assessment and development of maintenance and renewal schedules. The tool was commissioned by the division of Aurizon Holdings Ltd that owns, manages, and maintains, Australia's largest export coal rail network. The model provides a framework to minimize maintenance costs while ensuring high levels of reliability and capacity availability.

Boyles also explores the issue of accounting for user costs in the selection of maintenance projects. In the context of a road network, he explains that having costs increase in proportion to utilization levels favors projects for roads in urban areas. This, he argues, may result in (politically-unacceptable,) inequitable allocation of resources. In the paper, Boyles considers how preference for equitable distribution of benefits can be incorporated into alternative network-level maintenance optimization models, with some alternatives leading to intractable formulations. A numerical study is presented to compare the appealing specifications. The results 
show that considerable gains in equity are possible with only slight increases in total user cost.

We hope to have put together an issue that is enjoyable and useful to the journal's readership. To conclude, we express our gratitude to the authors who submitted papers, to the $25+$ reviewers who contributed their efforts to the refereeing process, and especially to the journal's Editor-in-Chief, Michel Bierlaire, and editorial staff. 